\title{
Análisis de los predictores psicosociales de la transgresión de normas de tráfico en España: los casos del uso del cinturón, del teléfono móvil, los límites de velocidad y el límite de alcoholemia al volante
}

Recibido el 23 marzo 2016/Publicado el 28 noviembre 2016

\author{
Rebeca Bautista Ortuño ${ }^{1}$ \\ Universidad Miguel Hernández de Elche \\ Esther Sitges Maciá ${ }^{2}$ \\ Universidad Miguel Hernández de Elche
}

\begin{abstract}
RESUMEN
El objetivo del presente trabajo fue determinar la influencia de variables derivadas de los enfoques de Disuasión, Influencia Social y Legitimidad en la transgresión de cuatro de las normas de tráfico cuyo incumplimiento más preocupa a autoridades y legisladores en España: el uso del cinturón de seguridad, la manipulación del teléfono móvil mientras se conduce, el exceso de velocidad y el exceso de alcoholemia al volante. Para alcanzar este objetivo, se administró una encuesta a 1500 conductores. Los modelos obtenidos muestran que la influencia social es un factor determinante para el cumplimiento de todas las normas de tráfico, que las variables de legitimidad tienen un peso importante para predecir la probabilidad de transgresión y que ninguno de los factores derivados del modelo de disuasión presentan influencia sobre estos comportamientos. Los resultados

\footnotetext{
${ }^{1}$ Autora responsable de la correspondencia: Centro CRÍMINA. Avda. de la Universidad s/n. 03202 Elche (Alicante). Dirección de e-mail: rbautista@umh.es

${ }^{2}$ Correspondencia: Centro CRÍMINA. Avda. de la Universidad s/n. 03202 Elche (Alicante). Dirección de e-mail: esther.sitges@umh.es
} 
sugieren una revisión de los planteamientos en los que se basan las políticas públicas de prevención en este contexto.

Palabras clave: Cumplimiento, Disuasión, Influencia social, Legitimidad, Seguridad vial

\begin{abstract}
The aim of this study was to determine the influence of variables derived from the approaches of Deterrence, Social Influence and Legitimacy in the violation of four traffic rules whose most worries to authorities and legislators in Spain: the use of seat belts, handling of the mobile phone while driving, speeding and driving while intoxicated by alcohol. To determine the role played by each factor in compliance with four of the most flouted traffic rules, a survey was administered by telephone to a random sample of 1500 Spanish drivers. The results obtained show the following conclusions: 1) social influence and legitimacy variables are determining factors to predict the probability of transgress every traffic rules; and 2) variables of the deterrence approach don't seems to influence the behavior. The results show the need for a review of current public policy approaches for prevention of dangerous driving behaviors.
\end{abstract}

Keywords: Compliance, Deterrence, Social Influence, Legitimacy, Road safety

\title{
1. Introducción
}

La prevención de la siniestralidad viaria en nuestro país sigue siendo una asignatura pendiente a juzgar por los datos arrojados en el último informe publicado por la Dirección General de Tráfico (DGT, 2015) al respecto. Aunque determinadas cifras como el número de víctimas mortales parecen haberse estabilizado y los accidentes de tráfico como causa de muerte externa han descendido del primer al quinto puesto en 2015 (INE, 2015), lo cierto es que existen datos preocupantes que indican que aún tenemos un largo camino por recorrer en el ámbito de la promoción de la seguridad vial. Según muestra el informe Las principales cifras de la siniestralidad vial (DGT, 2015), el número de accidentes con víctimas ha aumentado respecto a años anteriores y existe un alto porcentaje de conductores implicados en accidentes que habían cometido alguna infracción en vía interurbana (44\%) o en vía urbana (56\%). Además, el 58\% de las denuncias de la DGT fueron debidas a excesos de velocidad y el 30\% de los accidentes con víctimas se produjeron por distracciones, entre las cuales se encuentra la manipulación de dispositivos de telefonía móvil (DGT, 2015). A la luz de estos datos no es de extrañar 
que, con la mejora progresiva tanto de las vías como de la seguridad de los vehículos, la investigación científica haya puesto de manifiesto que actualmente la principal causa de la accidentalidad vial es el factor humano (Montoro, Alonso, Esteban y Toledo, 2000; Montoro, Roca y Tortosa, 2008; Tortosa y Civera, 2011), que engloba, entre otros comportamientos, la transgresión de las normas viales.

El estudio científico de la conducta humana ante las normas se ha abordado desde hace décadas desde diferentes enfoques, a partir de los cuales se defiende que la decisión de infringir o no un precepto formal está controlada por las consecuencias derivadas de la transgresión del mismo y que pueden ser de distinta naturaleza. Por ejemplo, desde la perspectiva del Modelo de la Disuasión (e.g. Paternoster, 2010), se defiende la importancia que tienen las características del castigo formal en la toma de decisiones y en el control conductual. Desde esta perspectiva se hipotetiza que, cuanto mayor sean la certeza, severidad y celeridad percibidas del castigo formal, menor será la probabilidad de que un individuo transgreda la ley. En cuanto a la certeza percibida, la mayor parte de estudios apoyan, aunque modestamente, la existencia de una relación entre la percepción de riesgo de ser sancionado y el incumplimiento informado (Lochner, 2007; Loughran, Pogarsky, Piquero y Paternoster, 2012; Matsueda, Kreager y Huizinga, 2006; Nagin, 1998; Paternoster, 1987). Por otra parte, y en relación a la severidad percibida, mientras que algunos autores han encontrado evidencias a favor de una relación inversa con la transgresión de las normas (Grasmick y Bryjak, 1980; Nagin y Pogarsky, 2001), otros investigadores afirman que sus efectos son poco consistentes (e.g. Paternoster, 1987). En lo que parece haber mayor acuerdo es en que la severidad percibida constituye un factor con menor impacto preventivo del que ejerce la certeza percibida (e.g. Nagin y Pogarsky, 2001). Por último, mientras que los teóricos clásicos otorgaron un papel fundamental a la contigüidad temporal del castigo para el fortalecimiento de la asociación entre la conducta desviada y sus consecuencias (Beccaria, 1764, 1969), estudios más recientes han puesto de manifiesto que, para algunos individuos, el coste que les supone una sanción demorada en el tiempo es mayor que si se les aplica rápidamente (Loewenstein, 1987). Algunos investigadores han llegado a afirmar incluso que la evidencia empírica no apoya la influencia de este factor sobre el comportamiento desviado de la norma (Nagin y Pogarsky, 2001). 
Tal y como se extrae del meta-análisis llevado a cabo por Pratt, Cullen, Blevins, Daigle y Madensen (2006), los efectos preventivos que ejercen las variables especificadas por el Modelo de la Disuasión sobre la conducta desviada son muy modestos. Como ya expresaron Meier, Burkett y Hickman (1984), no hay razones teóricas de peso que impidan extender los costes asociados a la transgresión de las normas a otros distintos a las meras sanciones formales que aplica el sistema legal. No obstante, a juzgar por las políticas públicas que viene desarrollando y aplicando el sistema, parece asumirse de partida que la clave para prevenir la transgresión radica en el incremento de los castigos formales, dejando de lado otros elementos que podrían ser más eficientes. La evidencia empírica sugiere que otros factores como, por ejemplo, las normas y sanciones sociales informales o el propio sistema de valores del individuo, pueden presentar también sólidos efectos preventivos (e.g. Anderson, Chiricos y Waldo, 1977; Bachman, Paternoster y Ward, 1992; Burkett y Ward, 1993; Grasmick y Green, 1980; Paternoster, 2010; Paternoster y Simpson, 1996).

Por lo que respecta a las sanciones sociales informales, el grueso de la investigación llevada a cabo en el ámbito de la influencia social pone de manifiesto el gran interés que, a lo largo de los años han mostrado los científicos sociales por las normas y su influencia sobre la conducta. Concretamente, y siguiendo los postulados de la Teoría Focal de la Conducta Normativa desarrollada por Cialdini, Reno y Kallgren (1990) y por Cialdini, Kallgren y Reno (1991), existen dos tipos de normas sociales que pueden influir en el comportamiento de los individuos. Por una parte, las denominadas normas descriptivas son las que se derivan de lo que los otros hacen, poniendo así de manifiesto el comportamiento que se considera típico o normal en un contexto determinado. Este tipo de información es más probable que opere en situaciones que son novedosas o ambiguas para el individuo (Sherif, 1936; Tesser, Campbell y Mickler, 1983), sobre todo cuando proviene de personas similares a él (Festinger, 1954). Por otra parte, las normas prescriptivas muestran aquello que es aprobado o no por la mayoría de la gente, es decir, aquello que se debería o no hacer; y, en definitiva, constituyen las reglas morales del grupo (Cialdini y Trost, 1998), señalando aquellos comportamientos que son impuestos por los demás a través de sanciones informales como la desaprobación social. 
Sin embargo, afirmar que las personas actúan condicionadas fundamentalmente por la amenaza de sanciones externas, formales o informales, constituye un razonamiento demasiado simplista para explicar la conducta humana en cualquier contexto. En la actualidad, las investigaciones desarrolladas por psicólogos sociales como Tom R. Tyler constituyen un marco de referencia respecto del estudio del cumplimiento de la ley desde la consideración de otros factores distintos al ejercicio del poder coercitivo, basado en la administración de castigos externos a modo de sanciones, tanto formales como informales, para motivar la obediencia y respeto de normas y autoridades. En opinión de Tyler (2009), las estrategias de control social en las que se basan la mayoría de estados resultan, cuanto menos, poco eficientes por dos razones. En primer lugar, el despliegue de recursos que harían falta para que la amenaza de castigo fuera percibida como real y disuadiera a los potenciales ofensores debería ser enorme. En segundo lugar, la literatura científica no arroja efectos preventivos consistentes del castigo formal, o bien, cuando se han encontrado son muy pequeños (McCoun, 1993; MacKenzie, 2002; Paternoster, 1987, 1989; Paternoster y Iovanni, 1986; Tyler, 2006b). Por estos motivos, el autor desarrolló el Modelo de la Autorregulación (Tyler, 2009), del que se extrae que la influencia de los valores personales en el comportamiento es mayor que la percepción de costes y beneficios externos que se esperan obtener de la conducta, $y$, además, que esos valores son capaces de motivar el cumplimiento voluntario de las normas así como la cooperación con las autoridades legales (Tyler, 2009). El valor central para este modelo es la legitimidad, definida como la cualidad que posee una autoridad, una ley o una institución, operativizándose como el apoyo mostrado hacia las autoridades legales, como el juicio moral de la norma y como el sentimiento de obligación hacia la obediencia de la ley, respectivamente. Algunos estudios manifiestan que el sistema de valores es importante para motivar el cumplimiento y, además, que su efecto es mayor que el que presentan las percepciones de riesgo de recibir un castigo formal (Sunshine y Tyler, 2003; Tyler, 2006b). La literatura muestra evidencias a favor de que es tal el poder de influencia de los valores sobre la conducta, que es menos probable que los individuos cumplan con normas que son consideradas inmorales o van en contra de su sistema de valores (Tyler, 2006b), al igual que es más probable que las cumplan si son coherentes con su moralidad (e.g. Carlsmith, Darley y Robinson, 2002; Gezelius, 2002; Tyler, 2006a). En este sentido, y en el ámbito de la seguridad vial, se ha encontrado, por ejemplo, que la probabilidad de 
transgredir el límite de alcoholemia al volante es inferior entre aquellos que consideran que esta norma es legítima que entre aquellos que piensan lo contrario (Tyler, Sherman, Strang, Barnes y Woods, 2007).

En nuestro país, los fenómenos de cumplimiento y transgresión de las normas en el ámbito de la seguridad vial se han abordado desde distintas perspectivas, aunque todas ellas con el objeto común de aumentar el conocimiento científico de estos comportamientos en aras de prevenir algunas de las causas más frecuentes de la siniestralidad como lo son las conductas irresponsables al volante. En este sentido, destacan algunos trabajos como el realizado por Alonso et al. (2005), un exhaustivo estudio descriptivo de las conductas de los conductores españoles y de las actitudes hacia las normas viales. Los resultados obtenidos mostraron, por una parte, que no existe una correspondencia entre las actitudes hacia las normas y el cumplimiento de las mismas. Otra de las conclusiones que se alcanzaron fue que no existe una elevada percepción de riesgo de ser sancionado por infringir los preceptos viales abordados. Por último, el estudio también puso de manifiesto que la peligrosidad percibida del comportamiento que prohíbe la norma, y la percepción de riesgo de sufrir un accidente si se lleva a cabo, son los factores más asociados al cumplimiento normativo.

Dado que, como se ha expuesto anteriormente, del Modelo de la Disuasión se extrae que los elementos clave para la prevención general negativa son la certeza, severidad y prontitud percibidas del castigo formal, otros grupos de investigación, por su parte, se han interesado en conocer qué variables psicológicas y de personalidad son las que determinan estas percepciones en el ámbito de la transgresión de las normas y las conductas peligrosas al volante. Por ejemplo, la investigación de Gómez-Fraguela y González-Iglesias (2010), que se llevó a cabo con una muestra de jóvenes conductoras, tipología muy poco estudiada en este ámbito, concluyó que determinadas variables de personalidad como la búsqueda de sensaciones y la ira están relacionadas con los comportamientos de riesgo en la conducción y, en concreto, con la transgresión de las normas de tráfico. En estudios realizados con muestras generales de conductores, por su parte, se ha puesto de manifiesto que, aunque los factores de personalidad por sí solos no tienen suficiente capacidad predictiva de conductas como la conducción en estado de embriaguez, otras variables individuales como las actitudes antisociales sí parecen ser 
determinantes de este tipo de comportamientos peligrosos durante la conducción (JornetGibert, Gallardo-Pujol, Suso y Andrés-Pueyo, 2013). Incluso se han desarrollado en nuestro país otras investigaciones centradas en analizar el perfil de los transgresores de determinadas normas de tráfico, tanto penales como no penales, pero en relación a la delincuencia común (Bautista y Miró-Llinares, 2014; Realpe y Serrano-Maíllo, 2015; Serrano-Maíllo y Realpe, 2015).

En cuanto a la modelización del cumplimiento normativo, existen otros trabajos que, por sus objetivos y metodología, han servido de referente para el estudio de los predictores de la obediencia a las normas en este contexto. Por ejemplo, los de Bautista (2012), Miró-Llinares y Bautista (2013) y Bautista, Sitges y Tirado (2015), llevan a cabo un análisis de los factores de distinta naturaleza asociados al cumplimiento de las normas de los límites de velocidad y del límite de alcoholemia al volante tras la reforma del Código Penal español en materia de seguridad vial. En dichos trabajos, realizados con muestras de conductores españoles, se han obtenido modelos en los que se refleja que los predictores más potentes del cumplimiento de estos preceptos viales son la influencia social y factores de legitimidad como el juicio moral de la norma. Resulta, por lo tanto, fundamental que, para fomentar el cumplimiento desde modelos alternativos a la amenaza de sanciones formales, tanto las normas como el comportamiento y decisiones de las autoridades legales reflejen en la mayor medida posible los estándares morales de la sociedad, compartidos por la mayoría de individuos.

Partiendo de los resultados de estos estudios previos, el presente trabajo se planteó con el objetivo de construir, con muestras representativas de conductores, modelos de regresión de la transgresión de normas viales. Estos modelos se dirigen a analizar la influencia conjunta ejercida por cada uno de los factores derivados de distintas tradiciones de investigación dentro del ámbito del cumplimiento normativo. Concretamente, el estudio se ha centrado en las normas referidas al uso del cinturón de seguridad, a la manipulación de dispositivos de telefonía móvil mientras se conduce, a los límites de velocidad y al límite de alcoholemia al volante, tipificadas en la Ley de Tráfico y Seguridad Vial 25/2009. Se eligieron estos cuatro preceptos debido a que por motivos de frecuencia y de peligrosidad, su incumplimiento es de los que más preocupan a autoridades y legisladores en el ámbito del tráfico y la seguridad vial. 
2. Método

\subsection{Participantes}

La muestra estuvo compuesta por 1500 conductores seleccionados de entre todas las Comunidades Autónomas de España mediante un procedimiento de muestreo aleatorio proporcional al tamaño de población, con cuota por ámbito geográfico, tamaño del hábitat (rural y urbano), sexo y edad. De los 1500 participantes, el $48 \%$ son hombres y el $52 \%$ son mujeres, de edades comprendidas entre los 18 y los 75 años $(M=44 ; D T=13,9)$.

\subsection{Variables}

En el estudio se evaluaron, como variables dependientes las relativas al incumplimiento informado de las normas abordadas: uso del cinturón de seguridad, conducción utilizando manualmente dispositivos de telefonía móvil, límites de velocidad y límite de alcoholemia. Como variables independientes se incluyeron las siguientes: 1) Conocimiento de la naturaleza (penal o administrativa) de las normas abordadas; 2) desde la perspectiva de la prevención general y especial negativa, variables pertenecientes al Modelo de la Disuasión, y 3) desde la perspectiva de la prevención general y especial positiva, variables del enfoque de Influencia Social y de Legitimidad. La definición operativa de todas ellas se muestra en la Tabla 1.

\section{Tabla 1}

Variables del estudio

\begin{tabular}{|c|c|c|}
\hline Incumplimiento & \multicolumn{2}{|c|}{ Frecuencia con la que los conductores infringen la norma } \\
\hline Conocimiento & \multicolumn{2}{|c|}{ Naturaleza administrativa vs penal de la norma } \\
\hline \multirow{4}{*}{ Disuasión } & \multirow{2}{*}{ Certeza } & Probabilidad percibida de ser sancionado \\
\hline & & Probabilidad percibida de tener un accidente/sufrir daños \\
\hline & Severidad & Sanción máxima percibida por incumplir la norma \\
\hline & Experiencia & $\mathrm{N}^{\mathrm{o}}$ de sanciones recibidas por transgresión de la norma \\
\hline \multirow{3}{*}{$\begin{array}{l}\text { Influencia } \\
\text { social }\end{array}$} & $\begin{array}{l}\text { Norma } \\
\text { prescriptiva }\end{array}$ & Desaprobación social percibida de la transgresión de la norma \\
\hline & \multirow{2}{*}{$\begin{array}{c}\text { Norma } \\
\text { descriptiva }\end{array}$} & Frecuencia percibida de incumplimiento por parte de la gente \\
\hline & & $\begin{array}{l}\text { Frecuencia percibida de incumplimiento por parte del } \\
\text { grupo de referencia }\end{array}$ \\
\hline \multirow{2}{*}{ Legitimidad } & \multicolumn{2}{|r|}{ Juicio moral de la norma } \\
\hline & \multicolumn{2}{|r|}{ Valoración de la adecuación del castigo formal } \\
\hline
\end{tabular}




\subsection{Instrumento}

Para la evaluación de todas las variables incluidas en el estudio se elaboró una encuesta ad hoc, basada en estudios anteriores de referencia como el de Tyler (2006b) realizado con una muestra de conductores estadounidenses, el de Oceja, Fernández-Dols, González, Jiménez y Berenguer (2001) con participantes chilenos, españoles y estadounidenses, y los trabajos de Bautista (2012), Miró-Llinares y Bautista (2013) y Bautista et al. (2015), en los que participaron conductores españoles.

Para el diseño de dicha encuesta se contó con los responsables de la Unidad de Investigación de la Dirección General de Tráfico, expertos en Derecho Penal, en Criminología, en Psicología y en Psicometría; y con antelación a su administración definitiva, se aplicó un estudio piloto previo para detectar y corregir errores de comprensión, antes de ser maquetada y administrada a la muestra de participantes del estudio.

\subsection{Procedimiento}

Las encuestas fueron administradas por el Sistema CATI (Computer Assisted Telephone Interview), a través del cual se efectuaron llamadas a números de teléfono tanto fijos como móviles. Cada encuesta tuvo una duración aproximada entre 12 y 15 minutos y, tras la recogida de datos mediante CATI, los ficheros fueron adaptados al programa SPSS para su posterior depuración y análisis.

Se llevó a cabo un análisis de regresión logística binaria múltiple para la construcción de los modelos mediante el método de pasos hacia delante basado en el estadístico de Wald. La razón principal por la que se eligió este método de construcción es la falta de modelos robustos previos que analicen de forma conjunta las variables abordadas en la transgresión de normas viales, y que podrían haber permitido priorizar la entrada de unos factores sobre otros a la hora de elaborarlos. La naturaleza de las variables y de los datos mostraron un mejor ajuste a este tipo de modelo, razón por la que se consideró que era la opción de análisis cuantitativo más adecuada para alcanzar los objetivos de la investigación. 


\section{Resultados}

Modelización de la transgresión de la norma del uso del cinturón de seguridad

Para evaluar la idoneidad del modelo de regresión se aplicaron las pruebas ómnibus sobre los coeficientes del modelo, cuyos resultados permiten rechazar la hipótesis nula de que los coeficientes de regresión de todos los términos incluidos en el modelo sean cero (Tabla 2). Finalmente, el coeficiente de determinación generalizado $\mathrm{R}^{2}$ de Nagelkerke presenta un valor de 0.131 tras la introducción de la última variable predictora seleccionada.

Partiendo de una probabilidad estimada umbral o de corte de $\mathrm{P}(\mathrm{Y}=1)=0.5$, el modelo permite una estimación correcta del 94\% de los casos.

\section{Tabla 2}

Pruebas de bondad de ajuste en cada paso

\begin{tabular}{|c|c|c|c|c|c|}
\hline & \multirow[t]{2}{*}{ Variables } & \multicolumn{3}{|c|}{ Pruebas ómnibus } & \multirow{2}{*}{$\begin{array}{c}\mathbf{R}^{2} \text { de } \\
\text { Nagelkerke }\end{array}$} \\
\hline & & $X^{2}$ & $\mathrm{gl}$ & $p$ & \\
\hline \multirow{2}{*}{ Paso 1} & Experiencia sanciones & 35.11 & 1 & 0.00 & \multirow[t]{2}{*}{0.064} \\
\hline & Modelo & 35.11 & 1 & 0.00 & \\
\hline \multirow{2}{*}{ Paso 2} & Adecuación castigo & 23.82 & 1 & 0.00 & \multirow[t]{2}{*}{0.107} \\
\hline & Modelo & 58.93 & 2 & 0.00 & \\
\hline \multirow{2}{*}{ Paso 3} & Norma descriptiva gente & 7.33 & 1 & 0.00 & \multirow[t]{2}{*}{0.120} \\
\hline & Modelo & 66.25 & 3 & 0.00 & \\
\hline \multirow{2}{*}{ Paso 4} & Norma descriptiva grupo & 6.72 & 1 & 0.01 & \multirow[t]{2}{*}{0.131} \\
\hline & Modelo & 72.97 & 4 & 0.00 & \\
\hline
\end{tabular}

Por último, se obtuvieron los coeficientes de regresión (B) de cada variable que ha entrado a formar parte del modelo junto con su error típico (E.T.), los valores y probabilidad asociada al estadístico de Wald y las odds ratio (OR) con sus intervalos de confianza (Tabla 3).

\section{Tabla 3}

Modelo de regresión logística para la probabilidad de incumplir la norma del uso de cinturón de seguridad 


\begin{tabular}{|ccccccc|}
\hline Modelo & B & E.T. & Wald & $\boldsymbol{p}$ & OR & IC 95\% para OR \\
\hline Experiencia sanciones & 3.992 & 0.817 & 23.898 & 0.000 & 54.156 & $10.929-268.353$ \\
\hline Adecuación castigo & -0.499 & 0.173 & 8.332 & 0.004 & 0.607 & $0.432-0.852$ \\
Norma descriptiva gente & 0.123 & 0.047 & 6.821 & 0.009 & 1.131 & $1.031-1.240$ \\
\hline Norma descriptiva grupo & 0.178 & 0.048 & 13.785 & 0.000 & 1.194 & $1.087-1.312$ \\
\hline
\end{tabular}

De la interpretación de los valores de las OD obtenidas se extrae que la probabilidad de infringir la norma del uso del cinturón: será menor en la medida en la que 1) el conductor considere que su transgresión debería sancionarse más severamente; y será mayor 2) cuanto mayor sea la frecuencia percibida de incumplimiento por parte de la gente en general, y 3 ) por parte del grupo de referencia. Por lo que respecta a la variable de experiencia con sanciones, la magnitud del error típico y la gran amplitud del intervalo de confianza de su OD sugieren que el valor del coeficiente de regresión obtenido no es fiable, por lo que debe interpretarse con cautela.

Modelización de la transgresión de la norma que prohíbe la manipulación de dispositivos de telefonía móvil mientras se conduce

Las pruebas ómnibus sobre los coeficientes del modelo indican que todos los correspondientes a las variables que entraron a formar parte del mismo son significativamente distintos a cero (Tabla 4). El modelo definitivo presenta un coeficiente de determinación generalizado moderado, ya que el valor de $\mathrm{R}^{2}$ de Nagelkerke es 0.168 .

\section{Tabla 4}

Pruebas de bondad de ajuste en cada paso

\begin{tabular}{|c|c|c|c|c|c|}
\hline & \multirow[t]{2}{*}{ Variables } & \multicolumn{3}{|c|}{ Pruebas ómnibus } & \multirow[t]{2}{*}{$\mathbf{R}^{2}$ de Nagelkerke } \\
\hline & & $X^{2}$ & $\mathrm{gl}$ & $p$ & \\
\hline \multirow{2}{*}{ Paso 1} & Conocimiento de la norma & 155.015 & 1 & 0.00 & \multirow[t]{2}{*}{0.139} \\
\hline & Modelo & 155.015 & 1 & 0.00 & \\
\hline \multirow{2}{*}{ Paso 2} & Adecuación castigo & 13.574 & 1 & 0.00 & \multirow[t]{2}{*}{0.151} \\
\hline & Modelo & 168.589 & 2 & 0.00 & \\
\hline \multirow{2}{*}{ Paso 3} & Desaprobación social & 9.021 & 1 & 0.00 & \multirow[t]{2}{*}{0.159} \\
\hline & Modelo & 177.610 & 3 & 0.00 & \\
\hline \multirow{2}{*}{ Paso 4} & Norma descriptiva grupo & 6.523 & 1 & 0.01 & \multirow[t]{2}{*}{0.164} \\
\hline & Modelo & 184.134 & 4 & 0.00 & \\
\hline \multirow{2}{*}{ Paso 5} & Juicio moral & 5.140 & 1 & 0.02 & \multirow[t]{2}{*}{0.168} \\
\hline & Modelo & 189.274 & 5 & 0.00 & \\
\hline
\end{tabular}


Con una probabilidad estimada de corte de $\mathrm{P}(\mathrm{Y}=1)=0.5$, el modelo permite una estimación correcta del $68.9 \%$ de los casos.

Una vez comprobado el ajuste del modelo, se obtuvieron los coeficientes de regresión, su significación y las OR (Tabla 5).

\section{Tabla 5}

Modelo de regresión logística para la probabilidad de incumplir la norma que prohíbe la manipulación de dispositivos de telefonía móvil mientras se conduce

\begin{tabular}{|ccccccc}
\hline Modelo & B & E.T. & Wald & $\boldsymbol{p}$ & OR & IC 95\% para OR \\
\hline Conocimiento de la norma & 0.341 & 0.130 & 6.912 & 0.009 & 1.406 & $1.091-1.813$ \\
\hline Adecuación castigo & -0.359 & 0.113 & 10.185 & 0.001 & 0.698 & $0.580-0.871$ \\
\hline Desaprobación social & 0.051 & 0.023 & 4.946 & 0.026 & 1.053 & $1.006-1.102$ \\
\hline Norma descriptiva grupo & 0.243 & 0.021 & 139.877 & 0.000 & 1.275 & $1.225-1.327$ \\
\hline Juicio moral & -0.144 & 0.045 & 10.301 & 0.001 & 0.866 & $0.793-0.946$ \\
\hline
\end{tabular}

Atendiendo a la interpretación de las OR se puede afirmar que la probabilidad de transgredir la norma que prohíbe la manipulación de dispositivos de telefonía móvil mientras se conduce será mayor si 1) el conductor tiene conocimiento de que la sanción por incumplirla es administrativa y no penal y 2) cuanto mayor sea la que frecuencia percibida de incumplimiento por parte del grupo de referencia. El factor desaprobación social, aunque ha entrado a formar parte del modelo, tiene un peso prácticamente nulo. Por otra parte, la probabilidad de transgresión de esta norma será menor 3) en la medida en que se considere que su transgresión debería sancionarse más severamente y 4) cuanto más se condene moralmente este comportamiento por parte del conductor.

\section{Modelización de la transgresión de los límites de velocidad}

Los resultados de las pruebas ómnibus acerca de los coeficientes del modelo indican que las variables que finalmente han entrado a formar parte del mismo presentan coeficientes de regresión significativos (Tabla 6). El tamaño del efecto alcanzado es moderado, con un valor de $\mathrm{R}^{2}$ de Nagelkerke de 0.182 . 


\section{Tabla 6}

Pruebas de bondad de ajuste en cada paso

\begin{tabular}{|c|c|c|c|c|c|}
\hline & \multirow[t]{2}{*}{ Variables } & \multicolumn{3}{|c|}{ Pruebas ómnibus } & \multirow[t]{2}{*}{$\mathbf{R}^{2}$ de Nagelkerke } \\
\hline & & $X^{2}$ & $\mathrm{gl}$ & $p$ & \\
\hline \multirow{2}{*}{ Paso 1} & Experiencia sanciones & 85.938 & 1 & 0.00 & \multirow[t]{2}{*}{0.080} \\
\hline & Modelo & 85.938 & 1 & 0.00 & \\
\hline \multirow{2}{*}{ Paso 2} & Severidad percibida & 45.071 & 1 & 0.00 & \multirow[t]{2}{*}{0.121} \\
\hline & Modelo & 131.010 & 2 & 0.00 & \\
\hline \multirow{2}{*}{ Paso 3} & Norma descriptiva grupo & 58.230 & 1 & 0.00 & \multirow[t]{2}{*}{0.171} \\
\hline & Modelo & 189.239 & 3 & 0.00 & \\
\hline \multirow{2}{*}{ Paso 4} & Juicio moral & 13.002 & 1 & 0.00 & \multirow[t]{2}{*}{0.182} \\
\hline & Modelo & 202.241 & 4 & 0.00 & \\
\hline
\end{tabular}

Con una probabilidad estimada de corte de $\mathrm{P}(\mathrm{Y}=1)=0.5$, el modelo permite una estimación correcta del $71.8 \%$ de los casos.

Posteriormente, se obtuvieron los coeficientes de regresión, su significación y las OR (Tabla 7).

\section{Tabla 7}

Modelo de regresión logística para la probabilidad de incumplir los límites de velocidad

\begin{tabular}{ccccccc}
\hline Modelo & B & E.T. & Wald & $\boldsymbol{p}$ & OR & IC 95\% para OR \\
\hline Experiencia sanciones & 1.342 & 0.226 & 35.297 & 0.000 & 3.826 & $2.458-5.957$ \\
Severidad percibida & 0.389 & 0.108 & 12.842 & 0.000 & 1.475 & $1.193-1.824$ \\
Norma descriptiva grupo & 0.167 & 0.020 & 67.792 & 0.000 & 1.182 & $1.006-1.102$ \\
Juicio moral & -0.188 & 0.032 & 34.554 & 0.000 & 0.828 & $0.778-0.882$ \\
\hline
\end{tabular}

Atendiendo a los resultados obtenidos del modelo, la probabilidad de infringir los límites de velocidad será mayor 1) cuanto mayor sea la severidad percibida del castigo, 2) cuanto mayor sea la frecuencia percibida de transgresión por parte del grupo de referencia y 3) cuanto menos se condene moralmente este comportamiento. Como ocurría en el primer modelo, los resultados respecto al factor relativo a haber sido sancionado en el pasado no son fiables debido al gran error típico de estimación obtenido.

\section{Modelización de la transgresión del límite de alcoholemia}

Las pruebas de bondad de ajuste indican que las variables que forman parte del modelo construido presentan valores significativamente distintos a cero (Tabla 8). 


\section{Tabla 8}

\section{Pruebas de bondad de ajuste en cada paso}

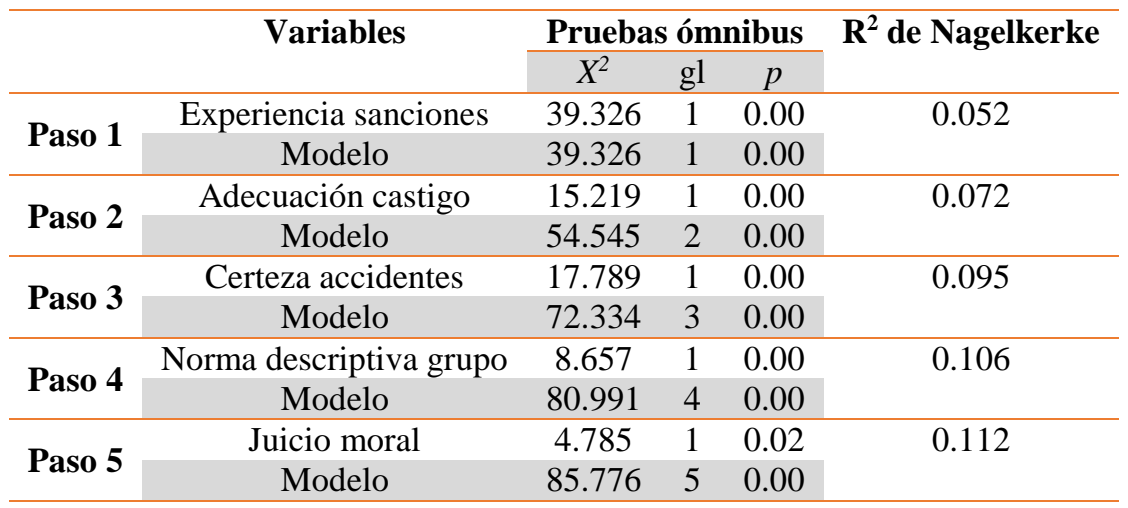

El tamaño del efecto del modelo alcanzó un valor de $\mathrm{R}^{2}$ de Nagelkerke de 0.112 y con una probabilidad estimada de corte de $\mathrm{P}(\mathrm{Y}=1)=0.5$; a partir de ello el modelo permite una estimación correcta del $88.7 \%$ de los casos.

\section{Tabla 9}

Modelo de regresión logística para la probabilidad de incumplir la norma del límite de alcoholemia

\begin{tabular}{ccccccc}
\hline Modelo & B & E.T. & Wald & $\boldsymbol{p}$ & OR & IC 95\% para OR \\
\hline Experiencia sanciones & 2.660 & 0.684 & 15.118 & 0.000 & 14.295 & $3.740-54.639$ \\
& & & & & & $0.411-0.774$ \\
Adecuación castigo & -0.572 & 0.161 & 12.593 & 0.000 & 0.564 & $0.897-0.993$ \\
Certeza accidentes & -0.058 & 0.026 & 5.027 & 0.025 & 0.943 & 0.897 \\
Norma descriptiva grupo & 0.215 & 0.034 & 38.974 & 0.000 & 1.240 & $1.159-1.326$ \\
Juicio moral & -0.157 & 0.053 & 8.673 & 0.003 & 0.855 & $0.770-0.949$ \\
\hline
\end{tabular}

La probabilidad de transgredir el límite de alcoholemia al volante será mayor 1) cuanto mayor sea la frecuencia percibida de transgresión por parte del grupo de referencia; y será menor 2) cuanto más severamente considere el conductor que debería castigarse su incumplimiento, 3) cuanto mayor sea la certeza percibida de sufrir un accidente de tráfico si se conduce bebido y 4) cuanto mayor sea la condena moral de este comportamiento. El modelo resultante también incluye, del mismo modo que dos de los tres expuestos anteriormente, la variable experiencia en el pasado con sanciones por transgredir esta norma; y, de igual forma que ocurría con los anteriores, la gran magnitud 
del error típico de estimación y la amplitud del intervalo de confianza de la OR indican que los resultados para este factor no son fiables.

\section{Discusión y conclusiones}

\section{Disuasión y transgresión de las normas}

Uno de los resultados más llamativos de entre los obtenidos radica en el hecho de que ni severidad ni certeza percibida del castigo formal han entrado a formar parte como variables predictoras de ninguno de los modelos construidos. Estos hallazgos están en consonancia con lo que apunta gran parte de la literatura científica que, desde hace décadas, está poniendo en duda los efectos disuasorios del castigo formal (e.g. Bautista y Miró-Llinares, 2015; Bautista et al., 2015; Miró-Llinares y Bautista, 2013; Paternoster, 2010). Cabe hacer mención al único efecto que se ha encontrado de un factor del enfoque de la disuasión como es la severidad percibida, que ha entrado a formar parte como variable predictora del modelo obtenido para pronosticar la probabilidad de transgresión de los límites de velocidad, pero en sentido contrario a lo que hipotetiza este enfoque. En otras palabras, cuanto más severas se perciben las sanciones que pueden ser aplicadas por exceso de velocidad, mayor es la probabilidad de llevar a cabo esta conducta. Para explicar este resultado podemos acudir a las hipótesis derivadas del enfoque de Legitimidad. Tratándose el exceso de velocidad de uno de los comportamientos al volante que de manera más frecuente es llevado a cabo por parte de los conductores, la percepción de que el castigo es más severo puede tener de base una consideración moral. Es decir, la idea de que las sanciones son altamente severas, combinada con la convicción de que se debería permitir conducir más rápido (Bautista, 2012; Miró-Llinares y Bautista, 2013), convierte al castigo formal en algo injusto cuanto más severo se perciba. Tal y como apunta Robinson (2012), "las personas que llegan a creer que los códigos legales se desvían de modo significativo de sus propios códigos morales sienten una preocupación menor respecto del cumplimiento de la ley" (p.202).

Estos resultados parecen ir en contra de lo esperado a partir de las intervenciones más importantes que, en el ámbito de la prevención general negativa, se llevan aplicando desde hace años por parte de legisladores y autoridades, para intentar reducir la accidentalidad viaria a partir de la motivación del cumplimiento normativo. Por otra parte, 
desde el punto de vista de la prevención especial, la baja prevalencia en la muestra de personas castigadas formalmente por transgredir las normas aquí abordadas ha impedido obtener resultados fiables y conclusiones definitivas en cuanto a la influencia de este factor. Concretamente, los porcentajes de conductores sancionados por no utilizar el cinturón de seguridad, por manipular dispositivos de telefonía móvil, por exceso de velocidad y por conducir en estado de embriaguez fueron $0.7 \%, 1.2 \%, 15.2 \%$ y $0.7 \%$ respectivamente. Por lo tanto, la baja variabilidad en este factor presente en nuestra muestra no ha permitido realizar estimaciones fiables de los coeficientes de regresión de la variable Experiencia con sanciones. Esta es, sin duda, una de las limitaciones de nuestro estudio, que pone de manifiesto la necesidad de llevar a cabo en el futuro investigaciones con muestras representativas de conductores previamente sancionados.

\section{Influencia Social y transgresión de las normas}

La influencia de la norma prescriptiva no ha sido lo suficientemente importante como factor preventivo de la probabilidad de infringir ninguna de las normas de tráfico abordadas. Cuando se analiza su influencia conjunta con otras variables, los resultados de los modelos construidos muestran que el comportamiento percibido por parte del grupo es el factor más estable y con coeficientes de regresión para cada uno de los modelos estimados con el menor error típico, siendo, por lo tanto, la variable predictora más fiable del incumplimiento. Estos resultados están en consonancia con lo que se ha observado en otros estudios, donde la norma descriptiva parece predecir mejor la conducta que la norma prescriptiva, cuando ambas están presentes y son igualmente accesibles (e.g. Bautista et al. 2015; Buunk y Bakker, 1995; Lewis y Neighbors, 2006; Stalans, Kinsey y Smith, 1991).

\section{Legitimidad y transgresión de las normas}

Con excepción de la conducta del uso del cinturón de seguridad, la probabilidad de transgredir el resto de preceptos viales aquí abordados depende también del juicio moral que realizan los sujetos de lo que prohíbe la norma. Es decir, cuanto más inaceptable se consideran los comportamientos como utilizar el teléfono móvil mientras se conduce, conducir bastante rápido y conducir bebido, menor es la probabilidad de transgresión de 
esas normas. Estos resultados, que son coherentes con los obtenidos en estudios anteriores (Bautista, 2012; Bautista et al., 2015), suponen una evidencia a favor del Modelo de la Autorregulación (Tyler, 2009) planteado en el inicio del presente artículo.

Por otra parte, otra de las variables derivadas del enfoque de la legitimidad, como la adecuación percibida del castigo, es otro de los factores que, con excepción del modelo relativo a los límites de velocidad, aparece de manera recurrente como variable predictora. Por lo tanto, y en consonancia con las hipótesis de partida, los conductores presentarán una probabilidad mayor de infringir las normas en la medida en la que consideren que las sanciones deberían ser más leves. Que este factor no haya entrado a formar parte del modelo relativo a la norma de los límites de velocidad se contrapone a lo obtenido en estudios anteriores con muestras de conductores españoles (Bautista et al., 2015; Bautista y Miró-Llinares, 2014; Miró-Llinares y Bautista, 2013). Los resultados arrojados en investigaciones previas muestran que la conducta al volante de los españoles parece depender más de la idea de que los límites de velocidad deberían ser menos estrictos, pese a considerar que conducir con exceso de velocidad no es un comportamiento adecuado (Alonso, Esteban, Calatayud y Sanmartín, 2013; Montoro, Roca y Lucas-Alba, 2010). Sin embargo, en el presente estudio el indicador relacionado con este factor que se ha evaluado, como es la valoración acerca de si el incumplimiento debería sancionarse más o menos severamente, no ha resultado significativo para la predicción del incumplimiento de esta norma.

A modo de conclusión, los resultados muestran la necesidad de una revisión de los planteamientos en los que actualmente se basan las políticas públicas en materia de prevención de los comportamientos peligrosos al volante. Se puede afirmar, por una parte, que el comportamiento de los conductores ante cada una de las normas de tráfico abordadas presenta diferencias que aconsejarían la diversificación de las estrategias de control social formal para fomentar los comportamientos responsables al volante, y lograr una mayor eficacia preventiva. Por otra parte, sin embargo, el estudio también ha permitido extraer algunos elementos que sí parecen claves para motivar el cumplimiento o, contrariamente, la transgresión de los preceptos formales abordados. La probabilidad de cumplir con las normas parece depender fundamentalmente de dos factores que son, por una parte, el comportamiento adoptado por el grupo de referencia, y, por otra, de que 
los preceptos formales se consideren moralmente aceptables. Sería conveniente, por lo tanto, que las normas formales impuestas y el trato proporcionado por las autoridades legales, se correspondiera con el sistema de valores compartido por los miembros de la sociedad.

\section{Agradecimientos}

El presente trabajo ha sido realizado en el marco del Proyecto de I+D+I titulado Análisis del cumplimiento efectivo de la legislación viaria y detección de factores de riesgo de la transgresión reiterada de normas de tráfico en España, con referencia SPIP2014-01294, financiado por la Dirección General de Tráfico.

\section{Referencias}

Alonso, F., Esteban, C., Calatayud, C., \& Sanmartín, J. (2013). Speed and Road Accidents: Behaviors, Motives, and Assessment of the Effectiveness of Penalties for Speeding. American Journal of Applied Psychology, 1(3), 58-64. doi: 10.12691/ajap-1-3-5

Alonso, F., Sanmartín, J., Calatayud, C., Esteban, C. Alamar, B., \& Ballestar, M. L. (2005). La justicia en el tráfico: Conocimiento y valoración de la población española. Barcelona: Attitudes.

Anderson, L. S., Chiricos, T. G., \& Waldo, G. P. (1977). Formal and informal sanctions: A comparison of deterrent effects. Social Problems, 25(1), 103-114. doi: $10.2307 / 800471$

Bachman, R., Paternoster, R., \& Ward, S. (1992). The rationality of sexual offending: Testing a deterrence/rational choice conception of sexual assault. Law and Society Review, 26(2), 343-372. doi: 10.2307/3053901

Bautista, R. (2012). La obediencia a las normas de tráfico: Predictores psicosociales del cumplimiento normativo tras la reforma del Código Penal de 2007 en materia de Seguridad Vial. Tesis doctoral no publicada. Universidad Miguel Hernández de Elche.

Bautista, R., \& Miró-Llinares, F. (2014). Delincuencia vial y reincidencia: un análisis de su prevalencia y concurrencia con la delincuencia común en la provincia de Alicante. Revista de Derecho Penal y Criminología, (11), 387-410.

Bautista, R., \& Miró-Llinares, F. (2015). ¿Por qué algunos siempre incumplen? Infractores y multi-infractores en seguridad vial. Indret: revista para el análisis del Derecho, (4), 1-38. 
Bautista, R., Sitges, E., \& Tirado, S. (2015). Psychosocial predictors of compliance with speed limits and alcohol limit by Spanish drivers: modeling compliance of traffic rules. Laws, 4(3), 602-616. doi: 10.3390/laws4030602

Beccaria. (1969). De los delitos y las penas (F. Tomás y Valiente, Trad.). Madrid: Aguilar. (Trabajo original publicado en 1764).

Burkett, S. R., \& Ward, D. A. (1993). A note of perceptual deterrence, religiously based moral condemnation, and social control. Criminology, 31(1), 119-134. doi: 10.1111/j.1745-9125.1993.tb01124.x

Buunk, B. P., \& Bakker, A. B. (1995). Extradyadic sex: the role of descriptive and injunctive norms. Journal of Sex Research, 32(4), 313-318. doi: $10.1080 / 00224499509551804$

Carlsmith, K. M., Darley, J. M., \& Robinson, P. H. (2002). Why do we punish? Deterrence and just deserts as motives for punishment. Journal of Personality and Social Psychology, 83(2), 284-299.

Cialdini, R. B., Kallgren, C. A., \& Reno, R. R. (1991). A focus theory of normative conduct: A theoretical refinement and reevaluation of the role of norms in human behavior. En L. Berkowitz (Ed.), Advances in experimental social psychology (pp. 201-234). San Diego: Academic Press.

Cialdini, R. B., Reno, R. R., \& Kallgren, C. A. (1990). A focus theory of normative conduct: Recycling the concept of norms to reduce littering in public places. Journal of Personality and Social Psychology, 58(6), 1015-1026. doi: 10.1037/0022-3514.58.6.1015

Cialdini, R. B., \& Trost, M. R. (1998). Social influence: social norms, conformity, and compliance. En D. T. Gilbert, S. T. Fiske \& G. Lindzey (Eds.), The handbook of social psychology (Vol. 2, pp. 151-192). Nueva York: McGraw-Hill.

Dirección General de Tráfico (2015). Las principales cifras de la siniestralidad vial. España 2014. Madrid: Dirección General de Tráfico.

Festinger, L. (1954). A theory of social comparison processes. Human Relations, 7(2), 117-140. doi: 10.1177/001872675400700202

Gezelius, S. S. (2002). Do norms count? State regulation and compliance in a Norwegian fishing community. Acta Sociologica, 45(4), 305-314. doi: 10.1177/000169930204500404

Gómez-Fraguela, J. A., \& Gónzález-Iglesias, B. (2010). El papel de la personalidad y la ira en la explicación de las conductas de riesgo al volante en mujeres jóvenes. Anales de Psicología, 26(2), 318-324.

Grasmick, H. G., \& Bryjak, G. J. (1980). The deterrent effect of perceived severity of punishment. Social Forces, 59(2), 471-491. doi: 10.1093/sf/59.2.471 
Grasmick, H. G., \& Green, D. E. (1980). Legal punishment, social dissaproval and internalization as inhibitors of illegal behavior. The Journal of Criminal Law and Criminology, 71(3), 325-335.

Instituto Nacional de Estadística (2015). Defunciones según la causa de muerte Notas de prensa. Recuperado de: http://www.ine.es/prensa/np896.pdf

Jornet-Gibert, M., Gallardo-Pujol, D., Suso, C., \& Andrés-Pueyo, A. (2013). Attitudes do matter: the role of attitudes and personality in DUI offenders. Accident Analysis and Prevention, 50, 445-450. doi: 10.1016/j.aap.2012.05.023

Lewis, M. A., \& Neighbors, C. (2006). Social norms approaches using descriptive drinking norms education: a review of the research on personalized normative feedback. Journal of American College Health, 54(4), 213-218. doi: 10.3200/JACH.54.4.213-218

Lochner, L. (2007). Individual perceptions of the criminal justice system. American Economic Review, 97(1), 444-460. doi: 10.1257/aer.97.1.444

Loewenstein, G. (1987). Anticipation and the valuation of delayed consumption. The Economic Journal, 97(387), 666-684.

Loughran, T. A., Pogarsky, G., Piquero, A. R., \& Paternoster, R. (2012). Reassessing the certainty effect in deterrence theory using insight from prospect theory. Justice Quarterly, 29, 712-741.

MacKenzie, D. L. (2002). Reducing the criminal activities of known offenders and delinquents: Crime prevention in the courts and corrections. En L.W. Sherman, D. P. Farrington, B. C. Welsh \& D. L. MacKenzie (Eds.), Evidence-based crime prevention (pp. 330-404). Londres: Routledge.

Matsueda, R. L., Kreager, D. A., \& Huizinga, D. (2006). Deterring delinquents: A rational choice model of theft and violence. American Sociological Review, 71(1), 95-122. doi: 10.1177/000312240607100105

McCoun, R. J. (1993). Drugs and the law: a psychological analysis of drug prohibition. Psychological Bulletin, 113(3), 497-512. doi: 10.1037//0033-2909.113.3.497

Meier, R. F., Burkett, S. R., \& Hickman, C. A. (1984). Sanctions peers, and deviance: Preliminary models of a social control process. The Sociological Quarterly, 25(1), 67-82. doi: 10.1111/j.1533-8525.1984.tb02239.x

Miró-Llinares, F., \& Bautista, R. (2013). ¿Por qué cumplimos las normas penales? Sobre la disuasión en materia de seguridad vial. InDret. Revista para el Análisis del Derecho, (4), 1-53.

Montoro, L., Alonso, F., Esteban, C., \& Toledo, F. (2000). Manual de seguridad vial: El factor humano. Barcelona: Ariel.

Montoro, L., Roca, J., \& Lucas-Alba, A. (2010). Creencias de los conductores españoles 
sobre la velocidad. Psicothema, 22(4), 858-864.

Montoro, L., Roca, J., \& Tortosa, F. (2008). Influence of the penalty point system on driving behaviour: drivers' point of view. Psicothema, 20(4), 652-658.

Nagin, D. S. (1998). Criminal deterrence research at the outset of the twenty-first century. Crime and Justice, 23, 1-42. doi: 10.1086/449268

Nagin, D. S., \& Pogarsky, G. (2001). Integrating celerity, impulsivity, and extralegal sanction threats into a model of general deterrence: Theory and evidence. Criminology, 39(4), 865-892. doi: 10.1111/j.1745-9125.2001.tb00943.x

Oceja, L. V., Fernández-Dols, J. M., González, A., Jiménez, I. \& Berenguer, J. (2001). ¿Por qué cumplimos las normas? Un análisis psicosocial del concepto de legitimidad. Revista de Psicología Social, 16(1), 21-41.

Paternoster, R. (1987). The deterrent effect of the perceived certainty and severity of punishment: a review of the evidence and issues. Justice Quarterly, 4(2), 173-217. doi: $10.1080 / 07418828700089271$

Paternoster, R. (1989). Decisions to participate in and desist from four types of common delinquency: Deterrence and the rational choice perspective. Law and Society Review 23(1), 7-40. doi: 10.2307/3053879

Paternoster, R. (2010). How much do really know about criminal deterrence? The Journal of Criminal Law and Criminology, 100(3), 765-824. doi: 10.2307/25766109

Paternoster, R. \& Iovanni, L. (1986). The deterrent effect of perceived severity: A reexamination. Social Forces 64(3), 751-77. doi: 10.1093/sf/64.3.751

Paternoster, R., \& Simpson, S. (1996). Sanction threats and appeals to morality: Testing a rational choice model of corporate crime. Law and Society Review, 30(3), 549584. doi: $10.2307 / 3054128$

Pratt, T. C., Cullen, F. T., Blevins, K. R., Daigle, L. E., \& Madensen, T. D. (2006). The empirical status of deterrence theory: A meta-analysis. En F. T. Cullen, J. P. Wright \& K. R. Blevins, Taking Stock. The status of criminological theory (pp. 367-395). Nueva Jersey: Transaction Publishers.

Realpe, M. F., \& Serrano-Maíllo, A. (2015). Infractores españoles en la conducción de vehículos a motor: ¿generalistas o especialistas? En F. Miró-Llinares, J. R. Agustina, J. E. Medina \& L. Summers (Eds.), Crimen, oportunidad y vida diaria: libro homenaje al profesor Dr. Marcus Felson (pp. 649-666). Madrid: Dykinson.

Robinson, P. H. (2012). Principios distributivos del Derecho Penal: a quién debe sancionarse y en qué medida. Madrid: Marcial Pons.

Serrano-Maíllo, A., \& Realpe, M. F. (2015). Criminología del delincuente vial. Madrid: B de F. 
Sherif, M. (1936). The psychology of social norms. Nueva York: Harper.

Stalans, L. J., Kinsey, K. A., \& Smith, K. V. (1991). Listening to different voices: formation of sanction beliefs and taxpaying norms. Journal of Applied Psychology, 21(2), 119-138. doi: 10.1111/j.1559-1816.1991.tb00492.x

Sunshine, J., \& Tyler, T. R. (2003). The role of procedural justice and legitimacy in shaping public support for policing. Law and Society Review, 37(3), 513-548. doi: $10.1111 / 1540-5893.3703002$

Tesser, A., Campbell, J., \& Mickler, S. (1983). The role of social pressure, attention to the stimulus, and self-doubt in conformity. European Journal of Social Psychology, 13(3), 217-233. doi: 10.1002/ejsp.2420130303

Tortosa, F., \& Civera, C. (2011). Es necesario controlar las conductas de consumo. Revista Española de Drogodependencias, 36(3), 245-248.

Tyler, T. R. (2006a). Psycohological perspectives on legitimacy and legitimation. Annual Review of Psychology, 57, 375-400. doi: 10.1146/annurev.psych.57.102904.190038

Tyler, T. R. (2006b). Why people obey the law. Princeton: Princeton University.

Tyler, T. R. (2009). Legitimacy and criminal justice: The benefits of self-regulation. Ohio State Journal of Criminal Law, 7, 307-359.

Tyler, T. R., Sherman, L. Strang, H., Barnes, G. C., \& Woods, D. (2007). Reintegrative shaming, procedural justice, and recidivism: The engagement of offenders' psychological mechanisms in the Canberra RISE drinking-and-driving experiment. Law and Society Review, 41(3), 553-586. doi: 10.1111/j.15405893.2007.00314.x 